\title{
THE SERIES EXPANSIONS AND GAUSS-LEGENDRE RULE FOR COMPUTING ARBITRARY DERIVATIVES OF THE BETA-TYPE FUNCTIONS*
}

\author{
JUNLIN LI ${ }^{\dagger}$, TONGKE WANG ${ }^{\dagger}$, AND YONGHONG HAO
}

\begin{abstract}
The beta-type functions play an important role in many applied sciences. The partial derivatives of the beta function and the incomplete beta function are integrals involving algebraic and logarithmic endpoint singularities. In this paper, some series expansions for these beta-type functions are found, which are easily used to evaluate these functions with prescribed precision. On the other hand, an accurate Gauss-Legendre quadrature formula is designed to compute these beta-type functions and their partial derivatives based on the Puiseux series for the integrands at their singularities. Some numerical examples confirm the high accuracy and high efficiency of the two algorithms, and also show that the algorithms can be used to effectively evaluate the generalized beta-type functions.
\end{abstract}

Key words. beta-type functions, higher-order partial derivatives, series expansion, modified Gauss-Legendre rule

AMS subject classifications. 65D20, 33B15, 33B20

1. Introduction. The beta-type functions play an important role in statistics and some other fields such as actuarial science, economics, finance, string theory, etc. The incomplete beta function is defined by the integral [10]

$$
B_{x}(p, q)=\int_{0}^{x} t^{p-1}(1-t)^{q-1} \mathrm{~d} t, \quad 0<x<1,
$$

where $p$ is a nonnegative real. The incomplete beta function involves a weakly singular integral when $0<p<1$. There are many efficient methods to compute the incomplete beta function. Didonato and Morris [3] calculated the incomplete beta function by using BRATIOa subroutine in FORTRAN. Ferreira et al. [5] derived a uniform convergent expansion of the incomplete beta function in terms of elementary functions. Temme [17, 18] and Doman [4] obtained some asymptotic expansions to approximate the incomplete beta function. Nemes et al. [9] also discussed its asymptotic expansion and gave a recurrence relation for the coefficients of the asymptotic expansion. According to [2], we know that the incomplete beta function can be written as a hypergeometric series, hence the proposed algorithm computes the approximations of the derivatives by differentiating the series.

The incomplete beta function degenerates to the beta function when $x=1$, that is,

$$
B(p, q)=\int_{0}^{1} t^{p-1}(1-t)^{q-1} \mathrm{~d} t .
$$

It is weakly singular when $0<p, q<1$ since the integrand has two singularities at $t=0,1$. In view of the relationship between the beta function and the gamma function, we can use the gamma function to compute the beta function. Besides, we can also compute the beta function by using the binomial expansion found in the papers by Osborn et al. [11] and Tang [16].

Some scholars have considered the partial derivatives of $B_{x}(p, q)$ [2]. The partial derivative of $(k, l)$ th-order of the incomplete beta function is obtained by differentiating (1.1) $k, l$

${ }^{*}$ Received April 16, 2019. Accepted January 29, 2020. Published online on April 3, 2020. Recommended by F. Marcellan. This project was supported by the Program for Innovative Research Team in Universities of Tianjin (TD13-5078) and the National Natural Science Foundation of China (grant No.11971241).

${ }^{\dagger}$ School of Mathematical Sciences, Tianjin Normal University, Tianjin 300387, P. R. China (wangtke@sina.com).

${ }^{\ddagger}$ Tianjin Key Laboratory of Water Resources and Environment, Tianjin Normal University, Tianjin 300387, P. R. China 
times with respect to $p, q$, respectively,

$$
B_{x}^{(k, l)}(p, q)=\frac{\partial^{k+l} B_{x}(p, q)}{\partial p^{k} \partial q^{l}}=\int_{0}^{x} t^{p-1}(1-t)^{q-1} \log ^{k} t \log ^{l}(1-t) \mathrm{d} t .
$$

Letting $x=1$ in $(1.3)$, the $(k, l)$ th-order partial derivative of the beta function is [1]

$$
B^{(k, l)}(p, q)=\frac{\partial^{k+l} B(p, q)}{\partial p^{k} \partial q^{l}}=\int_{0}^{1} t^{p-1}(1-t)^{q-1} \log ^{k} t \log ^{l}(1-t) \mathrm{d} t .
$$

In some applications, it is required to extend the arguments $p, q$ to negative numbers, which has been done by Özçag et al. in [12, 13]. They can also be extended to complex numbers [15]. In such cases, the integrals (1.1)-(1.4) do not exist in a usual sense, but they can be regularized to finite values in the neutrix sense $[12,13]$, or equivalently, in the Hadamard finite-part sense [6]. Hence, we call them generalized beta-type functions. In [14], the partial derivatives of the beta function were expressed in terms of a finite number of polygamma functions, and in [8], an algorithm for computing them was developed. In [7], some transformation properties of the incomplete beta function were derived, and the definitions of the partial derivatives of the incomplete beta function were extended to the whole complex plane.

As can be observed from formulas (1.1)-(1.2), we know that the beta-type functions are weakly $(0<p, q<1)$ or strongly $(p, q \leq 0)$ singular integrals at one or two endpoints. It will be shown later that the integral (1.3) or (1.4) is weakly singular when $0<l+p<1$ or strongly singular when $l+p \leq 0$. Considering these types of singular integrals, Wang et al. [20,21] designed a modified composite Gauss-Legendre rule to evaluate them by first expanding the integrands as Puiseux series at the singularities and then analyzing the asymptotic error expansions. We note that a Puiseux series is a generalization of the Taylor series that may contain negative and fractional exponents and logarithms.

In this paper, we first give a method to form the Puiseux series for the integrand at the singularities. By integrating the series term by term, we obtain series expansions for the beta-type functions in Section 2. We further use the Puiseux series to design a modified GaussLegendre rule to evaluate the beta-type functions and their partial derivatives in Section 3. We also extend the method to generalized beta-type functions in this section. In Section 4, some numerical tests are performed to illustrate the efficiency and accuracy of the series expansions and the numerical integration methods. We give a brief conclusion in Section 5 .

2. Series expansions for the partial derivatives of beta-type functions. For convenience, we define

$$
g_{1}(t)=t^{p-1} \log ^{k} t, \quad g_{2}(t)=(1-t)^{q-1} \log ^{l}(1-t), \quad \text { and } \quad f(t)=g_{1}(t) g_{2}(t) .
$$

In order to derive the series expansions for $f(t)$ at $t=0,1$, we need the following lemma:

LEMMA 2.1. For the function $\eta_{\alpha, m}(t)=t^{\alpha} \log ^{m} t$, where $\alpha$ is real and $m$ is an integer, the ith-order derivative of $\eta_{\alpha, m}(t)$ is $([19$, Lemma 6])

$$
\eta_{\alpha, m}^{(i)}(t)=t^{\alpha-i} \sum_{j=0}^{i}\left(\begin{array}{c}
m \\
j
\end{array}\right)(\log t)^{m-j} \sum_{\rho=0}^{i-j}(\rho+1)_{j} s(i, j+\rho) \alpha^{\rho},
$$

where $(\beta)_{j}$ is the standard Pochhammer symbol, defined by $(\beta)_{0}=1,(\beta)_{j}=\beta(\beta+1) \cdots$ $(\beta+j-1)$, and the $s(i, j)$ denote the Stirling numbers of the first kind defined by the recurrence relation [10] $s(i, j)=s(i-1, j-1)-(i-1) s(i-1, j)$ with initial values $s(0,0)=1$, $s(i, 0)=s(0, j)=0$, for $i, j>0$. 
Expanding $g_{2}(t)$ and $g_{1}(t)$ into a Taylor series at $t=0$ and $t=1$, respectively, and substituting them into $f(t)$, we have the following theorem:

THEOREM 2.2. The function $f(t)$ has the following expansions at $t=0,1$, respectively,

$$
\begin{aligned}
& f(t)=\sum_{i=0}^{\infty} c_{i}^{(1)}(q, l) t^{i+l+p-1} \log ^{k} t, \quad 0<t<1, \\
& f(t)=\sum_{i=0}^{\infty} c_{i}^{(2)}(p, k)(1-t)^{i+k+q-1} \log ^{l}(1-t), \quad 0<t<1,
\end{aligned}
$$

where

$$
\begin{aligned}
& c_{i}^{(1)}(q, l)=\frac{(-1)^{i+l}}{(i+l) !} \sum_{\rho=0}^{i}(\rho+1)_{l} s(i+l, l+\rho)(q-1)^{\rho}, \\
& c_{i}^{(2)}(p, k)=c_{i}^{(1)}(p, k), \quad i=0,1, \ldots
\end{aligned}
$$

Proof. Since the Taylor series for $(1-t)^{q-1}$ and $\log (1-t)$ at $t=0$ are both convergent for $0<t<1$, we can conclude that the Taylor series for $g_{2}(t)$ at $t=0$ is also convergent for $0<t<1$. Here we find another way to derive the series. Expanding $g_{2}(t)$ at $t=0$ and noting that $g_{2}^{(i)}(0)=(-1)^{i} \eta_{q-1, l}^{(i)}(1)$, we have

$$
g_{2}(t)=\sum_{i=0}^{\infty} \frac{g_{2}^{(i)}(0)}{i !} t^{i}=\sum_{i=0}^{\infty} \frac{(-1)^{i}}{i !} \eta_{q-1, l}^{(i)}(1) t^{i}, \quad 0<t<1 .
$$

From (2.2), we know that

$$
\eta_{\alpha, m}^{(i)}(1)= \begin{cases}0, & i<m \\ i-m & \\ \sum_{\rho=0}(\rho+1)_{m} s(i, m+\rho) \alpha^{\rho}, & i \geq m\end{cases}
$$

Hence,

$$
g_{2}(t)=\sum_{i=l}^{\infty} \frac{(-1)^{i}}{i !} \eta_{q-1, l}^{(i)}(1) t^{i}=\sum_{i=0}^{\infty} \frac{(-1)^{i+l}}{(i+l) !} \eta_{q-1, l}^{(i+l)}(1) t^{i+l}, \quad 0<t<1 .
$$

Substituting (2.6) into (2.7) and noting (2.1), we observe that (2.3) and (2.5) hold. Analogously, by expanding $g_{1}(t)$ into a Taylor series at the point $t=1$, we can show that (2.4) also holds. The theorem is proved.

Theorem 2.2 tells us that the partial derivative of $(k, l)$ th-order of the incomplete beta function (1.3) is strongly singular when $l+p \leq 0$ and weakly singular when $0<l+p<1$. For the case that $l+p \leq 0$, the incomplete beta function is interpreted as the Hadamard finite-part integral, usually denoted by $f_{0}^{x} f(t) \mathrm{d} t$. Integrating (2.3) over the interval $(0, x)$ term by term and using the integral identity [21]

$$
f_{0}^{x} t^{\alpha} \log ^{k} t \mathrm{~d} t= \begin{cases}x^{1+\alpha} \sum_{\rho=0}^{k} \frac{(-1)^{\rho}(k-\rho+1)_{\rho}}{(1+\alpha)^{\rho+1}}(\log x)^{k-\rho}, & \alpha \neq-1, \\ \frac{(\log x)^{k+1}}{k+1}, & \alpha=-1,\end{cases}
$$


we obtain the following theorem:

THEOREM 2.3. The partial derivative of $(k, l)$ th-order of the incomplete beta function has the following series expansion at $x$ :

$$
\begin{aligned}
B_{x}^{(k, l)}(p, q)= & \sum_{\substack{i=0 \\
i \neq-p-l}}^{\infty} c_{i}^{(1)}(q, l) x^{i+l+p} \sum_{\rho=0}^{k} \frac{(-1)^{\rho}(k-\rho+1) \rho}{(i+l+p)^{\rho+1}}(\log x)^{k-\rho} \\
& \quad+c_{-p-l}^{(1)}(q, l) \frac{(\log x)^{k+1}}{k+1}, \quad-p-l \geq 0,0<x<1 .
\end{aligned}
$$

REMARK 2.4. The formula (2.8) holds for every real $p$. When $p>0$, the formula is an expansion for the standard incomplete beta function at $x$, and the last term on the right-hand side of (2.8) vanishes. When $p \leq 0$, the formula is an expansion for the generalized incomplete beta function.

Analogously, integrating (2.4) over the interval $(x, 1)$ term by term yields

$$
\begin{gathered}
f_{x}^{1} f(t) \mathrm{d} t=\sum_{\substack{i=0 \\
i \neq-q-k}}^{\infty} c_{i}^{(2)}(p, k)(1-x)^{i+k+q} \sum_{\rho=0}^{l} \frac{(-1)^{\rho}(l-\rho+1)_{\rho}}{(i+k+q)^{\rho+1}}(\log (1-x))^{l-\rho} \\
\quad+c_{-q-k}^{(2)}(p, k) \frac{(\log (1-x))^{l+1}}{l+1}, \quad-q-k \geq 0,0<x<1 .
\end{gathered}
$$

The generalized beta function (1.4) can be split as

$$
B^{(k, l)}(p, q)=f_{0}^{1 / 2} f(t) \mathrm{d} t+f_{1 / 2}^{1} f(t) \mathrm{d} t
$$

Taking $x=\frac{1}{2}$ in (2.8), (2.9) and substituting them into (2.10), we obtain a practically useful series for evaluating the partial derivative of the generalized beta function

$$
\begin{aligned}
B^{(k, l)}(p, q)= & (-1)^{k} \sum_{\substack{i=0 \\
i \neq-p-l}}^{\infty} c_{i}^{(1)}(q, l)\left(\frac{1}{2}\right)^{i+l+p} \sum_{\rho=0}^{k} \frac{(k-\rho+1)_{\rho}}{(i+l+p)^{\rho+1}}(\log 2)^{k-\rho} \\
& +(-1)^{l} \sum_{\substack{i=0 \\
i \neq-q-k}}^{\infty} c_{i}^{(2)}(p, k)\left(\frac{1}{2}\right)^{i+k+q} \sum_{\rho=0}^{l} \frac{(l-\rho+1)_{\rho}}{(i+k+q)^{\rho+1}}(\log 2)^{l-\rho} \\
& +c_{-p-l}^{(1)}(q, l) \frac{(-\log 2)^{k+1}}{k+1}+c_{-q-k}^{(2)}(p, k) \frac{(-\log 2)^{l+1}}{l+1}, \\
& -p-l \geq 0,-q-k \geq 0 .
\end{aligned}
$$

In order to accelerate the convergence of the series (2.8) when $x>\frac{1}{2}$, we compute the partial derivative of the generalized incomplete beta function using the formula

$$
B_{x}^{(k, l)}(p, q)=f_{0}^{1} f(t) \mathrm{d} t-f_{x}^{1} f(t) \mathrm{d} t
$$

and the two integrals are expanded as the series (2.11) and (2.9), respectively.

Since the coefficients in (2.5) are given explicitly, the series (2.8), (2.9), (2.11), and (2.12) can be evaluated with as many terms as necessary with respect to the index $i$ such that the 
remainder term is less than $10^{-16}$. For this purpose, we have written a Mathematica function Abet $a[x, p, q, k, 1]$ to calculate the derivatives of all the beta-type functions by a series expansions, where $x$ is the upper limit of the incomplete beta function and $p, q, k, l$ are the parameters in (1.3) or (1.4). If $x=1$, the algorithm automatically evaluate the complete beta-type functions.

3. The modified Gauss-Legendre rule for beta-type functions and their partial derivatives. In this section, we develop an efficient Gauss-Legendre rule to uniformly evaluate beta-type functions.

3.1. Review of the error expansion of the Gauss-Legendre rule for weakly singular integrals. Let $f(t)$ be sufficiently smooth in $(a, b)$. We can expand it as Puiseux series at $t=a$ or $t=b$ or both of them. That is, $f(t)$ has the following asymptotic expansions

$$
\begin{aligned}
& f(t)=\sum_{i=0}^{u} \sum_{j=0}^{u_{i}} c_{i, j}^{(1)}(t-a)^{\alpha_{i}}(\log (t-a))^{\mu_{i, j}}+r_{a}(t):=f_{a}(t)+r_{a}(t), \quad t>a \\
& f(t)=\sum_{i=0}^{v} \sum_{j=0}^{v_{i}} c_{i, j}^{(2)}(b-t)^{\beta_{i}}(\log (b-t))^{\nu_{i, j}}+r_{b}(t):=f_{b}(t)+r_{b}(t), \quad t<b
\end{aligned}
$$

where $u, u_{i}, v, v_{i}, \mu_{i, j}, \nu_{i, j}$ are all nonnegative integers and $\alpha_{i}, \beta_{i}$ are all real numbers satisfying $-1<\alpha_{0}<\alpha_{1}<\cdots<\alpha_{u},-1<\beta_{0}<\beta_{1}<\cdots<\beta_{v}$. It is noted that the remainders $r_{a}(t), r_{b}(t)$ can be made sufficiently smooth on $[a, b]$ by choosing $u, v$ suitably large.

Remark 3.1. The Puiseux series (3.1) can be generated by the Series command of Mathematica. By writing Series $[f[t],\{t, a, u\}]$, where $u$ is a given integer, we can completely determine $u_{i}, \mu_{i, j}$, and $c_{i, j}^{(1)}$ in (3.1). We also know the asymptotic behaviour of the remainder, namely $r_{a}(t)=o\left((t-a)^{u}\right)$. Usually, it is difficult to discuss the convergence of the series (3.1), and hence it is hardly possible to obtain the integral value by integrating (3.1) term by term. What we need to do is design some other methods to evaluate the integral of $f(t)$ using the singular expansions (3.1) or (3.2). We note that the series (2.3) is a special case of (3.1), which has been shown to be convergent for $0<t<1$, and hence we obtain a convergent series (2.8) by integrating (2.3) term by term.

For a function $g(t) \in C(0,1)$, we define a general $r$-point Gauss-Legendre formula

$$
\int_{0}^{1} g(t) \mathrm{d} t \approx \sum_{\lambda=1}^{r} \sigma_{\lambda} g\left(\theta_{\lambda}\right)
$$

where $\sigma_{\lambda}>0$ and $\theta_{\lambda} \in(0,1)(\lambda=1,2, \ldots, r)$ are the weights and abscissas. We note that the standard $r$-point Gauss-Legendre quadrature is defined on the interval $[-1,1]$, where the abscissas are the zeros of the Legendre polynomial $P_{r}(x)$, denoted by $\theta_{\lambda}^{*}, \lambda=1,2, \ldots, r$. The corresponding weights are obtained by $\sigma_{\lambda}^{*}=2 /\left(\left(1-\theta_{\lambda}^{* 2}\right)\left(P_{r}^{\prime}\left(\theta_{\lambda}^{*}\right)\right)^{2}\right)$. In this paper, we transform the sampling points into $[0,1]$, and hence $\theta_{\lambda}=\left(1+\theta_{\lambda}^{*}\right) / 2$ and $\sigma_{\lambda}=\sigma_{\lambda}^{*} / 2$. One advantage of the Gauss-Legendre rule is that all the abscissas are located in the interval $(0,1)$, and hence we do not have to treat the endpoint singularities in a special way for the singular integral. The Gauss-Legendre rule has the highest degree of accuracy, which means that it can be used to integrate polynomials of degree $2 r-1$ exactly using only $r$ nodes.

We consider the computation of the definite integral $I=\int_{a}^{b} f(t) \mathrm{d} t$. Divide $[a, b]$ into $n$ equal subintervals with step length $h=(b-a) / n$ and the nodes denoted by $t_{i}=a+i h$, 
where $i=0,1, \ldots, n$. Then by (3.3), we obtain a composite Gauss-Legendre rule

$$
I=\sum_{i=0}^{n-1} \int_{t_{i}}^{t_{i+1}} f(t) \mathrm{d} t \approx h \sum_{\lambda=1}^{r} \sigma_{\lambda} \sum_{i=0}^{n-1} f\left(a+\left(i+\theta_{\lambda}\right) h\right):=Q_{n} .
$$

The error remainder of the formula is denoted by

$$
E_{n}=I-Q_{n} .
$$

In the following lemma, we present the asymptotic expansion of the error with step length $h$ for the Gauss-Legendre quadrature formula when the integrand $f(t)$ is singular at the point $t=a$ or $t=b$ or both of them.

Lemma 3.2 ([20, Theorem 3.2, (4.10)]). Suppose that $f(t) \in C(a, b)$ is sufficiently smooth except at the endpoint $t=a$, where around this point, $f(t)$ is assumed to have the Puiseux expansion (3.1). In this case, the integral remainder $E_{n}$ (here denoted by $R_{1}$ ) of the quadrature formula $Q_{n}$ can be expressed as an asymptotic expansion with respect to $h$,

$$
\begin{aligned}
R_{1}=-\sum_{i=0}^{u} & \sum_{j=0}^{u_{i}} c_{i, j}^{(1)} h^{1+\alpha_{i}} \sum_{m=0}^{\mu_{i, j}}\left(\begin{array}{c}
\mu_{i, j} \\
m
\end{array}\right)(\log h)^{\mu_{i, j}-m} \\
& \times\left(\sum_{\lambda=1}^{r} \sigma_{\lambda} \theta_{\lambda}^{\alpha_{i}}\left(\log \theta_{\lambda}\right)^{m}-\frac{(-1)^{m} m !}{\left(1+\alpha_{i}\right)^{m+1}}\right)+\cdots
\end{aligned}
$$

For the case that $f(t)$ has the Puiseux expansion (3.2), the integral remainder $E_{n}$ (here denoted by $R_{2}$ ) reads

$$
\begin{aligned}
R_{2}=- & \sum_{i=0}^{v} \sum_{j=0}^{v_{i}} c_{i, j}^{(2)} h^{1+\beta_{i}} \sum_{m=0}^{\nu_{i, j}}\left(\begin{array}{c}
\nu_{i, j} \\
m
\end{array}\right)(\log h)^{\nu_{i, j}-m} \\
& \times\left(\sum_{\lambda=1}^{r} \sigma_{\lambda} \theta_{\lambda}^{\beta_{i}}\left(\log \theta_{\lambda}\right)^{m}-\frac{(-1)^{m} m !}{\left(1+\beta_{i}\right)^{m+1}}\right)+\cdots
\end{aligned}
$$

For the case that $f(t)$ has the Puiseux expansions (3.1) and (3.2) at $t=a$ and $t=b$, respectively, the integral remainder $E_{n}$ is simply the sum of (3.5) and (3.6), that is, $E_{n}=R_{1}+R_{2}$.

We note that only the main part of the error is written in (3.5) or (3.6), and the omitted part is smaller than the main part; see reference [20] for the complete expression of the error. Since the Puiseux expansions (3.1) or (3.2) can be handled by symbolic computation, the main part of the integral remainder (3.5) or (3.6) is computable. Hence, we can add one or both of them to the Gauss-Legendre rule (3.4) to correct the accuracy of the quadrature formula.

3.2. Modified Gauss-Legendre rule for partial derivatives of beta-type functions. For the incomplete beta function (1.1) and its $(k, l)$ th-order derivative (1.3), the integrand $f(t)$ in (2.1) is singular at $t=0$ when $l+p<1$. Hence, by truncating the series (2.3), keeping only finitely many terms like (3.1), and taking $n=1, h=x, \alpha_{i}=i+l+p-1, \mu_{i, j}=k$ in the formulas (3.4), (3.5), we obtain a modified Gauss-Legendre rule for computing the $(k, l)$ th-order partial derivative of the incomplete beta function by adding the main part of the error in (3.5) to (3.4)

$$
\begin{aligned}
B_{x}^{(k, l)}(p, q) & \approx x \sum_{\lambda=1}^{r} \sigma_{\lambda} f\left(\theta_{\lambda} x\right)-\sum_{i=0}^{u} c_{i}^{(1)}(q, l) x^{i+l+p} \\
& \times \sum_{m=0}^{k}\left(\begin{array}{c}
k \\
m
\end{array}\right)(\log x)^{k-m}\left(\sum_{\lambda=1}^{r} \sigma_{\lambda} \theta_{\lambda}^{i+l+p-1} \log ^{m} \theta_{\lambda}-\frac{(-1)^{m} m !}{(i+l+p)^{m+1}}\right),
\end{aligned}
$$


with leading error term

$$
\begin{aligned}
e_{u}=-c_{u}^{(1)} & (q, l) x^{u+l+p} \sum_{m=0}^{k}\left(\begin{array}{c}
k \\
m
\end{array}\right)(\log x)^{k-m} \\
& \times\left(\sum_{\lambda=1}^{r} \sigma_{\lambda} \theta_{\lambda}^{u+l+p-1} \log ^{m} \theta_{\lambda}-\frac{(-1)^{m} m !}{(u+l+p)^{m+1}}\right) .
\end{aligned}
$$

When $x=1$, the incomplete beta function degenerates to the complete beta function, which is singular at both $t=0,1$ when $0<p, q<1$. For such case, we truncate the series (2.3), (2.4) at a finite index like (3.1), (3.2), respectively. By taking $n=1, h=1, \alpha_{i}=i+l+p-1$, $\mu_{i, j}=k, \beta_{i}=i+k+q-1, \nu_{i, j}=l$ in the formulas (3.4), (3.5), and (3.6), we obtain a modified Gauss-Legendre rule for evaluating the $(k, l)$ th-order derivative of the beta function

$$
\begin{gathered}
B^{(k, l)}(p, q) \approx \sum_{\lambda=1}^{r} \sigma_{\lambda} f\left(\theta_{\lambda}\right)-\sum_{i=0}^{u} c_{i}^{(1)}(q, l)\left(\sum_{\lambda=1}^{r} \sigma_{\lambda} \theta_{\lambda}^{i+l+p-1} \log ^{k} \theta_{\lambda}-\frac{(-1)^{k} k !}{(i+l+p)^{k+1}}\right) \\
-\sum_{i=0}^{v} c_{i}^{(2)}(p, k)\left(\sum_{\lambda=1}^{r} \sigma_{\lambda} \theta_{\lambda}^{i+k+q-1} \log ^{l} \theta_{\lambda}-\frac{(-1)^{l} l !}{(i+k+q)^{l+1}}\right)
\end{gathered}
$$

with leading error term

$$
\begin{aligned}
e= & -c_{u}^{(1)}(q, l)\left(\sum_{\lambda=1}^{r} \sigma_{\lambda} \theta_{\lambda}^{u+l+p-1} \log ^{k} \theta_{\lambda}-\frac{(-1)^{k} k !}{(u+l+p)^{k+1}}\right) \\
& -c_{v}^{(2)}(p, k)\left(\sum_{\lambda=1}^{r} \sigma_{\lambda} \theta_{\lambda}^{v+k+q-1} \log ^{l} \theta_{\lambda}-\frac{(-1)^{l} l !}{(v+k+q)^{l+1}}\right) .
\end{aligned}
$$

It can be seen from (3.7) that the formula for computing the partial derivative of the beta function is especially simple and hence has high efficiency. It is noted that the incomplete beta function is nearly singular at $t=x$ when $x$ is close to 1 , which will deteriorate the computational accuracy. In order to increase the computational accuracy, we introduce a constant $\delta \in(0.5,1)$. When $x \geq \delta$, the formula for computing the incomplete beta function becomes

$$
B_{x}^{(k, l)}(p, q)=\int_{0}^{1} f(t) \mathrm{d} t-\int_{x}^{1} f(t) \mathrm{d} t:=I_{1}-I_{2},
$$

where $I_{1}$ is the complete beta function, which can be evaluated by (3.7) and $I_{2}$ is singular at the point $t=1$, which can be calculated by the following Gauss-Legendre rule. Taking $n=1$, $h=1-x, \beta_{i}=i+k+q-1, \nu_{i, j}=l$ in the formulas (3.4), (3.6), we obtain a modified Gauss-Legendre rule for $I_{2}$ by adding the main part of the error in (3.6) to (3.4)

$$
\begin{aligned}
I_{2} \approx(1 & -x) \sum_{\lambda=1}^{r} \sigma_{\lambda} f\left(x+\theta_{\lambda}(1-x)\right)-\sum_{i=0}^{v} c_{i}^{(2)}(p, k)(1-x)^{i+k+q} \\
& \times \sum_{m=0}^{l}\left(\begin{array}{c}
l \\
m
\end{array}\right)(\log (1-x))^{l-m}\left(\sum_{\lambda=1}^{r} \sigma_{\lambda} \theta_{\lambda}^{i+k+q-1} \log ^{m} \theta_{\lambda}-\frac{(-1)^{m} m !}{(i+k+q)^{m+1}}\right),
\end{aligned}
$$


with leading error term

$$
\begin{aligned}
e_{v}=-c_{v}^{(2)} & (p, k)(1-x)^{v+k+q} \sum_{m=0}^{l}\left(\begin{array}{c}
l \\
m
\end{array}\right)(\log (1-x))^{l-m} \\
\times & \left(\sum_{\lambda=1}^{r} \sigma_{\lambda} \theta_{\lambda}^{v+k+q-1} \log ^{m} \theta_{\lambda}-\frac{(-1)^{m} m !}{(v+k+q)^{m+1}}\right) .
\end{aligned}
$$

3.3. Extension to generalized beta-type functions. We know from (2.3) and (2.4) that when $l+p \leq 0$ or $k+q \leq 0$, the beta-type functions (1.3), (1.4) are defined in the sense of Hadamard finite-part integrals. We denote them as generalized beta-type functions. The key point to evaluate the Hadamard finite-part integral is to transform it to a regular one by separating the singular part. For example, for the generalized incomplete beta function, by denoting

$$
f_{0}(t)=\sum_{i=0}^{w} c_{i}^{(1)}(q, l) t^{i+l+p-1} \log ^{k} t
$$

where $w$ is chosen such that $w+l+p-1 \geq 0$, we have

$$
\begin{aligned}
f_{0}^{x} f(t) \mathrm{d} t= & f_{0}^{x} f_{0}(t) \mathrm{d} t+\int_{0}^{x}\left(f(t)-f_{0}(t)\right) \mathrm{d} t \\
= & \sum_{\substack{i=0 \\
i \neq-p-l}}^{w} c_{i}^{(1)}(q, l) x^{i+l+p} \sum_{\rho=0}^{k} \frac{(-1)^{\rho}(k-\rho+1)_{\rho}}{(i+l+p)^{\rho+1}}(\log x)^{k-\rho} \\
& +c_{-p-l}^{(1)}(q, l) \frac{(\log x)^{k+1}}{k+1}+\int_{0}^{x}\left(f(t)-f_{0}(t)\right) \mathrm{d} t, \quad-p-l \geq 0 .
\end{aligned}
$$

For the regular integral in (3.9), we can effectively evaluate it using the modified GaussLegendre rule in Section 3.2.

For the generalized beta function (1.4), since the points $t=0,1$ are the strong singularities of $f(t)$ when $l+p \leq 0$ and $k+q \leq 0$, we only need to split the integral as

$$
f_{0}^{1} f(t) \mathrm{d} t=f_{0}^{1 / 2} f(t) \mathrm{d} t+f_{1 / 2}^{1} f(t) \mathrm{d} t
$$

and then employ the above method to evaluate the two integrals on the right-hand side of (3.10); see [21] for details.

At the end of this section, we consider issues of practical computation. We should first set the computational accuracy, then fix the values of $r, u, v$, and $\delta$. By repeated testing, we could achive a computational accuracy of $10^{-16}$ by taking $r=19, u=6, v=6$. Furthermore, we take $\delta=0.82$ in the formula (3.8) or (3.9). Using these settings, we have written a Mathematica function to implement the algorithm, Dbet $a[x, p, q, k, 1]$, where $x$ is the upper limit of the incomplete beta function and $p, q, k, l$ are the parameters in (1.3) or (1.4). We note that the weights and abscissas of the Gauss-Legendre rule can be obtained by the Mathematica command NIntegratèGaussBernt senEspelidRuleData. If $x=1$, then the algorithm automatically implements the formula (3.7) or (3.10) to evaluate the partial derivative of the beta function. In particular, when $k=l=0$ it evaluates the beta function as well. If $0<x<1$, then the partial derivative of the incomplete beta function is computed by the algorithm. It can also compute the incomplete beta function when $k=l=0$. Since the coefficients of the series expansions for $f(t)$ at $t=0$ and $t=1$ are derived from (2.5), the algorithm can be easily implemented on other software platforms. 


\section{ETNA}

Kent State University and

Johann Radon Institute (RICAM)

4. Numerical results. In this section, we perform some experiments to show that both the series expansion (Abeta) and the modified Gauss-Legendre rule (Dbeta) are capable of evaluating various beta-type functions. The computational results are shown in Tables 4.14.4, where C-value and T-error represent the computational value and the absolute true error, respectively. We note that a more accurate value is obtained by Mathematica with 20 significant digits, and the difference of this and the computational value yields the T-error. However, sometimes the values can not be evaluated by Mathematica, in which case the T-error is indicated by the symbol "-".

TABLE 4.1

The results of Abeta and Dbet a for computing the derivatives of the incomplete beta function.

\begin{tabular}{l|ll|ll}
\multirow{2}{*}{$(x, p, q, k, l)$} & \multicolumn{3}{|c}{ Abeta } & \multicolumn{3}{c}{ Dbeta } \\
\cline { 2 - 5 } & C-value & T-error & C-value & T-error \\
\hline$\left(\frac{2}{5}, \frac{1}{30}, \frac{1}{700}, 0,0\right)$ & 29.578154453855 & $3.55 \mathrm{E}-15$ & 29.578154453855 & $1.52 \mathrm{E}-16$ \\
$\left(\frac{2}{5}, \frac{7}{109}, \frac{27}{32}, 3,1\right)$ & 4.8151823956904 & $8.88 \mathrm{E}-16$ & 4.8151823956904 & $5.40 \mathrm{E}-16$ \\
$\left.\frac{13}{25}, \frac{7}{10}, \frac{9}{30}, 2,1\right)$ & -0.501645429170 & $1.11 \mathrm{E}-16$ & -0.501645429170 & $8.98 \mathrm{E}-17$ \\
$\left(\frac{17}{35}, \frac{3}{7}, \frac{1}{9}, 0,0\right)$ & 2.0257081264806 & $4.44 \mathrm{E}-16$ & 2.0257081264806 & $4.34 \mathrm{E}-16$ \\
$\left(\frac{2}{5}, \frac{6}{27}, \frac{9}{115}, 2,1\right)$ & -1.217155071073 & $2.22 \mathrm{E}-17$ & -1.217155071073 & $1.42 \mathrm{E}-16$ \\
$\left.\frac{9}{10}, \frac{37}{15}, \frac{67}{409}, 2,3\right)$ & -0.169013488180 & $1.11 \mathrm{E}-16$ & -0.169013488180 & $1.90 \mathrm{E}-16$ \\
$\left(\frac{21}{25}, \frac{7}{30}, \frac{4}{5}, 2,3\right)$ & -0.191480573558 & $1.67 \mathrm{E}-16$ & -0.191480573558 & $5.00 \mathrm{E}-16$ \\
$\left.\frac{22}{25}, \frac{19}{4}, \frac{32}{67}, 2,3\right)$ & -0.047635921875 & $6.94 \mathrm{E}-17$ & -0.047635921875 & $2.08 \mathrm{E}-17$ \\
$\left(\frac{11}{25}, \frac{1}{7}, \frac{1}{200}, 0,0\right)$ & 6.6821089021104 & $8.88 \mathrm{E}-16$ & 6.6821089021104 & $8.88 \mathrm{E}-16$ \\
$\left(\frac{40}{89}, \frac{7}{36}, \frac{1}{7}, 0,0\right)$ & 4.7689183458079 & $2.66 \mathrm{E}-15$ & 4.7689183458079 & $8.88 \mathrm{E}-16$ \\
$\left(\frac{47}{96}, \frac{1}{5}, \frac{33}{67}, 2,1\right)$ & -1.294161981407 & $2.22 \mathrm{E}-16$ & -1.294161981407 & $2.22 \mathrm{E}-16$ \\
$\left(\frac{77}{120}, \frac{7}{16}, \frac{1}{3}, 2,1\right)$ & -0.886443726519 & $1.11 \mathrm{E}-16$ & -0.886443726519 & $1.11 \mathrm{E}-16$ \\
$\left(\frac{103}{150}, \frac{1}{2}, \frac{1}{3}, 0,1\right)$ & -0.777549461512 & $3.00 \mathrm{E}-15$ & -0.777549461512 & $1.11 \mathrm{E}-16$
\end{tabular}

TABLE 4.2

The results of Abeta and Dbet a for computing the derivatives of the beta function.

\begin{tabular}{l|ll|ll}
\multirow{2}{*}{$(p, q, k, l)$} & \multicolumn{3}{|c}{ Abeta } & \multicolumn{3}{c}{ Dbeta } \\
\cline { 2 - 5 } & C-value & T-error & C-value & T-error \\
\hline$\left(\frac{1}{3}, \frac{7}{2}, 0,0\right)$ & 1.82208690692024 & $2.22 \mathrm{E}-16$ & 1.82208690692024 & $4.36 \mathrm{E}-16$ \\
$\left.\frac{23}{15}, \frac{1}{7}, 1,3\right)$ & 3.50233254395884 & $4.44 \mathrm{E}-16$ & 3.50233254395884 & $7.75 \mathrm{E}-15$ \\
$\left.\frac{1}{20}, \frac{12}{5}, 0,0\right)$ & 18.8327157668963 & $3.55 \mathrm{E}-15$ & 18.8327157668963 & $2.67 \mathrm{E}-15$ \\
$\left.\frac{6}{7}, \frac{8}{11}, 0,3\right)$ & -21.554718266433 & $1.07 \mathrm{E}-14$ & -21.554718266433 & $1.23 \mathrm{E}-14$ \\
$\left.\frac{1}{29}, \frac{1}{600}, 8,6\right)$ & 0.01653514722849 & $1.32 \mathrm{E}-16$ & 0.01653514722849 & $8.95 \mathrm{E}-16$ \\
$\left.\frac{27}{30}, \frac{7}{9}, 5,7\right)$ & 0.01082138659110 & $1.54 \mathrm{E}-16$ & 0.01082138659110 & $6.64 \mathrm{E}-17$ \\
$\left(\frac{13}{9}, \frac{2}{3}, 5,5\right)$ & 0.01083909103055 & $1.11 \mathrm{E}-16$ & 0.01083909103055 & $5.25 \mathrm{E}-18$ \\
$\left(\frac{1}{5}, \frac{9}{4}, 1,3\right)$ & 0.10613958415905 & $6.94 \mathrm{E}-17$ & 0.10613958415905 & $1.93 \mathrm{E}-16$ \\
$\left(\frac{33}{12}, \frac{33}{700}, 2,4\right)$ & 0.53224115177277 & $1.11 \mathrm{E}-16$ & 0.53224115177277 & $2.84 \mathrm{E}-16$ \\
$\left.\frac{13}{16}, \frac{7}{8}, 4,1\right)$ & -1.3316102792395 & $2.22 \mathrm{E}-16$ & -1.3316102792395 & $6.00 \mathrm{E}-16$ \\
$\left(\frac{17}{3}, \frac{8}{7}, 2,5\right)$ & -0.0633663704157 & $9.71 \mathrm{E}-17$ & -0.0633663704157 & $3.53 \mathrm{E}-17$ \\
$\left(\frac{19}{7}, \frac{3}{23}, 2,1\right)$ & -0.4355635721872 & $3.33 \mathrm{E}-16$ & -0.4355635721872 & $2.06 \mathrm{E}-15$ \\
$\left.\frac{158}{23}, \frac{12}{99}, 7,9\right)$ & 0.00051973938302 & $1.32 \mathrm{E}-16$ & 0.00051973938302 & $3.85 \mathrm{E}-19$ \\
$\left(\frac{258}{12}, \frac{7}{17}, 7,9\right)$ & 0.00001681198242 & $7.39 \mathrm{E}-17$ & 0.00001681198242 & $1.71 \mathrm{E}-14$
\end{tabular}


TABLE 4.3

The results of Abeta and Dbet a for computing the derivatives of the generalized incomplete beta function.

\begin{tabular}{|c|c|c|c|c|}
\hline \multirow{2}{*}{$(x, p, q, k, l)$} & \multicolumn{2}{|c|}{ Abeta } & \multicolumn{2}{|c|}{ Dbeta } \\
\hline & C-value & T-error & C-value & T-error \\
\hline & -18.097083037 & - & -18.097083037 & - \\
\hline & 40.89521976165 & - & 40.89521976165 & - \\
\hline 2,1$)$ & -104.45569477 & - & -104.45569477 & - \\
\hline$\left.\frac{1}{800}, 0,2\right)$ & -7.0473728308 & $8.88 \mathrm{E}-16$ & -7.0473728308 & $4.44 \mathrm{E}-15$ \\
\hline$, 1,2)$ & -21.328866949 & $1.42 \mathrm{E}-14$ & -21.328866949 & $7.82 \mathrm{E}-14$ \\
\hline & -19.574914940 & & & \\
\hline$\left.-\frac{7}{8}, 1,2\right)$ & -41.3 & & & \\
\hline$\left.-\frac{3}{8}, 1,1\right)$ & 102.7781640080 & $4.26 \mathrm{E}-14$ & 102. & -14 \\
\hline$\left.-\frac{57}{90}, 1,3\right)$ & 201.7129058196 & $2.84 \mathrm{E}-14$ & 201.7129058196 & -13 \\
\hline$\left.-\frac{21}{8},-\frac{1}{7}, 0,1\right)$ & -1.2750175805 & & -1.27 & -14 \\
\hline$\left.,-\frac{7}{4}, \frac{5}{8}, 1,2\right)$ & -17.615540613 & & -17.6 & E-13 \\
\hline$\left(\frac{19}{20},-\frac{1}{8},-\frac{5}{300}, 1,0\right)$ & -65.997189610 & $4.26 \mathrm{E}-14$ & -65.997189610 & $1.67 \mathrm{E}-16$ \\
\hline$\left(\frac{93}{100},-\frac{39}{9}, \frac{15}{6}, 0,2\right)$ & 0.342694637011 & $6.11 \mathrm{E}-16$ & 0.342694637011 & $1.23 \mathrm{E}-13$ \\
\hline$\left.-\frac{1}{6},-\frac{1}{4}, 1,0\right)$ & -38.676112897 & $7.11 \mathrm{E}-15$ & -38.676112897 & $1.42 \mathrm{E}-14$ \\
\hline
\end{tabular}

TABLE 4.4

The results of Abeta and Dbet a for computing the derivatives of the generalized beta function.

\begin{tabular}{l|ll|ll}
\multirow{2}{*}{$(p, q, k, l)$} & \multicolumn{3}{|c}{ Abeta } & \multicolumn{2}{c}{ Dbeta } \\
\cline { 2 - 5 } & C-value & T-error & C-value & T-error \\
\hline$(-5,-2,0,0)$ & -29.45 & $5.33 \mathrm{E}-14$ & -29.44999999996 & $4.10 \mathrm{E}-11$ \\
$(-7,-2,2,1)$ & -99.55630679348 & - & -99.55630679642 & - \\
$(-1,-4,3,3)$ & 61.851513452306 & - & 61.851513452305 & - \\
$\left(\frac{5}{2},-\frac{4}{9}, 0,1\right)$ & -0.369927140833 & $6.66 \mathrm{E}-16$ & -0.369927140833 & $5.05 \mathrm{E}-15$ \\
$\left(-\frac{7}{3},-\frac{1}{2}, 0,2\right)$ & 43.235104435821 & $7.11 \mathrm{E}-15$ & 43.235104435821 & $5.47 \mathrm{E}-13$ \\
$\left(-\frac{21}{9},-\frac{7}{8}, 1,1\right)$ & 7.4766169400929 & $8.88 \mathrm{E}-16$ & 7.4766169400929 & $1.07 \mathrm{E}-14$ \\
$\left(-\frac{11}{5},-\frac{1}{5}, 1,1\right)$ & 49.624447731140 & $2.84 \mathrm{E}-14$ & 49.624447731140 & $4.26 \mathrm{E}-14$ \\
$\left(-\frac{4}{3},-\frac{7}{6}, 1,0\right)$ & 11.596191137200 & $1.07 \mathrm{E}-14$ & 11.596191137200 & $7.11 \mathrm{E}-15$ \\
$\left(-\frac{33}{10},-\frac{17}{9}, 1,2\right)$ & -1.747593695331 & $2.22 \mathrm{E}-16$ & -1.747593695331 & $3.71 \mathrm{E}-14$ \\
$\left(-\frac{7}{9},-\frac{5}{6}, 1,0\right)$ & -46.96657258136 & $1.42 \mathrm{E}-14$ & -46.96657258136 & $1.42 \mathrm{E}-14$ \\
$\left(-\frac{15}{8},-\frac{5}{2}, 0,0\right)$ & 54.717940661539 & $7.11 \mathrm{E}-15$ & 54.717940661538 & $8.24 \mathrm{E}-13$ \\
$\left(-\frac{17}{6}, \frac{25}{4}, 0,1\right)$ & -52.88581961244 & $2.84 \mathrm{E}-14$ & -52.88581961244 & $7.11 \mathrm{E}-15$ \\
$\left(-\frac{10}{3},-\frac{9}{8}, 2,3\right)$ & 8.4189198647365 & $3.55 \mathrm{E}-15$ & 8.4189198647370 & $5.08 \mathrm{E}-13$ \\
$\left(-\frac{15}{4},-\frac{16}{7}, 1,2\right)$ & 132.01970328127 & $5.68 \mathrm{E}-14$ & 132.01970328127 & $1.82 \mathrm{E}-12$
\end{tabular}

Tables 4.1, 4.3 exhibit some results for the incomplete beta function and the generalized incomplete beta function, and Tables 4.2, 4.4 show the results for the beta function and the generalized beta function, respectively. The computation indicates that series expansion and numerical integration are both accurate for evaluating beta-type functions. In order to achieve a precision of $10^{-16}$, the series expansion in (2.3) or (2.4) has to include up to 50 terms in some cases, but the numerical integration only requires its first 6 terms. Certainly, the numerical integration needs to evaluate the integral by the Gauss-Legendre rule. In practical computation, both of them are feasible. 
5. Conclusion. The beta-type functions can be evaluated by many efficient numerical or symbolic methods. In this paper, we form the Puiseux series for the integrand at its singularities, which play twofold roles in this paper. First, we derive series expansion for the incomplete beta function at the upper limit and then use it to evaluate the beta-type functions. Second, we present a modified Gauss-Legendre integration method to uniformly treat the beta-type functions and their derivatives by using the Puiseux series. These two algorithms are accurate and efficient, which is illustrated by calculating some typical examples.

Acknowledgments. The authors are grateful to the editors and referees for their valuable comments and suggestions.

\section{REFERENCES}

[1] F. AL-SiRehy AND B. Fisher, Further results on the beta function and the incomplete beta function, Appl. Math. Sci. (Ruse), 7 (2013), pp. 3489-3495.

[2] R. J. Boik And J. F. Robison-Cox, Derivatives of the incomplete beta function, J. Stat. Softw., 3 (1999), pp. 1-19.

[3] A. R. Didonato AND A. H. MorRis, Algorithm 708: significant digit computation of the incomplete beta function ratios, ACM. Trans. Math. Software, 18 (1992), pp. 360-373.

[4] B. G. S. Doman, An asymptotic expansion for the incomplete beta function, Math. Comp., 65 (1996), pp. 1283-1288.

[5] C. Ferreira, J. L. López, AND E. PÉRez Sinusía, Uniform representations of the incomplete beta function in terms of elementary functions, Electron. Trans. Numer. Anal., 48 (2018), pp. 450-461. http://etna.mcs.kent.edu/vol.48.2018/pp450-461.dir/pp450-461.pdf

[6] J. Hadamard, Lectures on Cauchy's Problem in Linear Partial Differential Equations, Dover Publications, New York, 1953.

[7] A. LI AND H. QIN, Some transformation properties of the incomplete beta function and its partial derivatives, IAENG Int. J. Appl. Math., 49 (2019), pp. 109-113.

[8] A. LI, Z. SUN, AND H. QIN, The algorithm and application of the beta function and its partial derivatives, Engr. Lett., 23 (2015), pp. 140-144.

[9] G. NemES AND A. B. OlDE DAALHUIS, Uniform asymptotic expansion for the incomplete beta function, SIGMA Symmetry Integrability Geom. Methods Appl., 12 (2016), Art. 101, 5 pages.

[10] F. W. J. Olver, D. W. Lozier, R. F. Boisvert, AND C. W. Clark, NIST Handbook of Mathematical Functions, Cambridge University Press, Cambridge, 2010.

[11] D. OsBoRn AND R. MADEY, The incomplete beta function and its ratio to the complete beta function, Math. Comp., 22 (1968) pp. 159-162.

[12] E. ÖZÇAḠ, I. EGE, AND H. GÜRÇAY, An extension of the incomplete beta function for negative integers, J. Math. Anal. Appl., 338 (2008), pp. 984-992.

[13] E. ÖZÇAḠ, I. Ege, H. GÜRÇAY, AND B. Jolevska-Tuneska, On partial derivatives of the incomplete beta function, Appl. Math. Lett., 21 (2008), pp. 675-681.

[14] N. Shang, A. Li, Z. SUn, AND H. QIN, A note on the beta function and some properties of its partial derivatives, IAENG Int. J. Appl. Math., 44 (2014), pp. 200-205.

[15] Z. SUN, H. QIN, AND A. LI, Extension of the partial derivatives of the incomplete beta function for complex values, Appl. Math. Comput., 275 (2016), pp. 63-71.

[16] I. C. TANG, On the computation of a certain type of incomplete beta functions, Commun. ACM, 6 (1963), p. 689.

[17] N. M. TEMME, Uniform asymptotic expansions of the incomplete gamma functions and the incomplete beta function, Math. Comp., 29 (1975), pp. 1109-1114.

[18] _- Asymptotic inversion of the incomplete beta function, J. Comput. Appl. Math., 41 (1992), pp. 145-157.

[19] T. WANG, Y. GU, AND Z. ZHANG, An algorithm for the inversion of Laplace transforms using Puiseux expansions, Numer. Algorithms, 78 (2018), pp. 107-132.

[20] T. WANG, Z. LIU, AND Z. ZHANG, The modified composite Gauss type rules for singular integrals using Puiseux expansions, Math. Comp., 86 (2017), pp. 345-373.

[21] T. WANG, Z. ZHANG, AND Z. LIU, The practical Gauss type rules for Hadamard finite-part integrals using Puiseux expansions, Adv. Comput. Math., 43 (2017), pp. 319-350. 